\title{
Goods or resource contests?
}

\author{
Martin Kolmar
}

Received: 12 December 2001 / Accepted: 21 December 2006 / Published online: 25 January 2007

(C) Springer Science + Business Media B.V. 2007

\begin{abstract}
In this paper we analyze the optimal mix between appropriative and defensive activities for goods (goods contest) and appropriative and defensive activities for resources (resource contest). We show that goods contests are qualitatively different from resource contests. It turns out that for plausible cases the resource contest is dominated by the goods contest in the sense that investments are only positive in the latter.
\end{abstract}

Keywords Conflict $\cdot$ Anarchy $\cdot$ Goods $\cdot$ Resources

\section{JEL Classification: D74}

\section{Introduction}

The growing literature on anarchy and conflict initiated by Bush and Mayer (1974) analyzes the structure equilibria of different allocation problems in the presence of appropriative and defensive activities. Most models of appropriation and defense analyze either a contest over resources or a contest over goods. Examples for the first class of models are de Meza and Gould (1992), Grossman and Kim (1995), Grossman (2001), and Hirshleifer (1995). All papers analyze allocation problems where agents are endowed with a productive resource that can be appropriated. This resource can be used for the production of a final good which itself is unalienable. Grossman (2001) for example analyzes a model of self-enforced property rights where agents can appropriate and defend a resource necessary for the production of a final good. Once produced, however, every agent consumes the whole amount of his production. Examples for the second class of models are Grossman (2002), Grossman and Kim (2000), Skaperdas (1992), Skaperdas and Syropoulos (1995). Skaperdas and Syropoulos (1997) for example analyze a situation where individuals can either invest in production or appropriation, where the resources are unalienable but the final goods are due to appropriation.

\section{Kolmar}

Institute for Empirical Economics and Economic Policy, University of St. Gallen, Varnbüelstrasse 14, CH-9000 St. Gallen, Switzerland e-mail:martin.kolmar@unisg.ch 
Economic models that analyze resource and goods contests separately have the same formal structure. In a resource contest it makes no formal difference if one defines the utility of an agent on the consumption goods or directly on the final distribution of resources. In the latter case a mapping from resources to utility has to be defined that has the same properties as the sequential mapping from resources into production and from production into consumption. With such a mapping both problems are equivalent and one can interpret the resources as consumption goods of the 'new' utility function. ${ }^{1}$ The terms 'goods' or 'resource contest' are therefore only a matter of interpretation without referring to any underlying structural difference.

This formal analogy shows that the distinctive features of goods and resource contests, if there are any, can only be analyzed in a model that includes both contests simultaneously. We show that a combination of both contests reveals that there exists such a qualitative difference between the appropriation of goods and the appropriation of resources: goods contests are a more direct way to increase utility because the effect of the appropriation of resources that increases own production is 'filtered' by the outcome of the goods contest. This filter works as follows. First, an increase in the production of an agent is only rational for this agent if some of the goods produced can be defended against appropriation. Otherwise an increase in resources is irrelevant. Second, an increase in the production of one agent resulting from a reallocation of resources reduces the production of the other agent. If an agent is very successful if appropriating goods, an increase in resources can decrease his utility because of this effect. None of this effects is relevant for a goods contest. An increase in appropriation of goods unambiguously increases the utility of the agent.

Depending on the economic problem to be analyzed the restriction to either a resource or goods contest can be appropriate. For some goods the act of consumption cannot be separated from the act of production, which implies that the goods are unalienable. By the same token some resources may be unalienable because of their characteristics. Excluding the possibility of slavery, time is a good example for such a resource. However, in a situation of anarchy it in general possible that agents can and will appropriate both, goods and resources, and the question arises how resources will be devoted to both contests. This paper analyzes the interrelation between the appropriation and defense of goods and the appropriation and defense of resources. We characterize conditions for which the restriction to investments in either only the resource or in only the goods contest are an equilibrium of the contest. In this sense we provide a microfoundation for the restriction to either of the two forms of contests in situations where in principle resources as well as goods are due to appropriation.

The latter remark points to a limitation of the analysis. Because resources are needed to appropriate, it is impossible that all resources may be appropriated. There must exist at least one resource that is used for appropriation and that is itself unalienable. Otherwise the problem is not meaningfully specified. We assume that such a resource exists and call it time.

Most of the literature cited above restricts attention to the analysis of Tullock contest functions and some form of generalized Cobb-Douglas production function. One of the main findings of this paper is that in such an economy the resource contest is dominated by the goods contest as long as the agents are sufficiently homogenous. The agents will not invest in the resource contest but spend only resources for the appropriation of goods. This result shows that the restriction to resource contests is a critical assumption in the sense that this restriction cannot be derived from a model where the agents have the choice of appropriative investments. Resource contests in Tullock-Cobb-Douglas economies are

\footnotetext{
${ }^{1}$ See Lancaster (1977) for the idea of a household production function and the formal details. 
therefore a meaningful tool only in situations where the characteristics of the goods make them unalienable. By the same token, the restriction to goods contests can easier be justified as the outcome of a more complicated model.

The paper proceeds as follows. In Section 2 we present the general model. In Section 3 we discuss two functional specifications of the model. Section 4 concludes.

\section{The model}

We analyze an economy that is populated by two economic agents indexed by $i=1,2$. Both agents have an endowment of one unit of time and one unit of another productive resource. Every agent can spend his time for six different activities. He can spend $l_{i}$ units of time for the production of a final good whose quantity is given by $y_{i}$ and $f_{i}$ units of time for leisure. The final good is produced by the use of time and the resource,

$$
y_{i}=F_{i}\left(l_{i}, r_{i}\right),
$$

with positive but decreasing marginal productivities and strictly convex isoquants.

Time is not due to appropriation by the other agent but capital and the final good. Hence, the agents can spend $a_{i}^{r}$ units of time to appropriate part of the resource of the other agent and $a_{i}^{y}$ units of time to appropriate part of the good produced by the other agent. Accordingly, $d_{i}^{r}$ and $d_{i}^{y}$ denote the time investments to defend against the appropriation of the resource and the final good by the other agent.

We denote by $\pi_{i}^{r}\left(d_{i}^{r}, a_{j}^{r}\right)$ and $\pi_{i}^{y}\left(d_{i}^{y}, a_{j}^{y}, \theta\right)$ the fraction of the resource and the fraction of the good not appropriated by the other agent given investment levels $d_{i}^{r}, a_{j}^{r}$, and $d_{i}^{y}, a_{j}^{y}$. $\theta$ denotes a parameter that measures differences in the effectiveness of appropriative and defensive activities between the resource and the goods contest. The contest-success functions have the following properties:

$$
\begin{aligned}
& \pi_{i}^{x}\left(0, a_{j}^{x}\right)=0 \quad \text { if } a_{j}^{x}>0, \quad \pi_{i}^{x}\left(d_{i}^{x}, 0\right)=0 \quad \text { if } d_{i}^{x}>0, \\
& \frac{\partial \pi_{i}^{x}}{\partial d_{i}^{x}}>0, \quad \frac{\partial \pi_{i}^{x}}{\partial a_{j}^{x}}<0, \quad \frac{\partial^{2} \pi_{i}^{x}}{\partial\left(d_{i}^{x}\right)^{2}}<0, \quad \frac{\partial^{2} \pi_{i}^{x}}{\partial\left(a_{j}^{x}\right)^{2}}>0, \quad x \in\{r, y\} .
\end{aligned}
$$

These properties are for example fulfilled by the Tullock function discussed in the next section.

The resource constraint of agent $i$ is

$$
1=l_{i}+a_{i}^{r}+d_{i}^{r}+a_{i}^{y}+d_{i}^{y}+f_{i},
$$

and the resulting allocation of the resource is

$$
r_{i}=\pi_{i}^{r} \cdot 1+\left(1-\pi_{j}^{r}\right) \cdot 1
$$

Accordingly, the final consumption of agent $i$ of $\operatorname{good} i$ and $j, c_{i}^{i}, c_{i}^{j}$, is equal to

$$
c_{i}^{i}=\pi_{i}^{y} y_{i}, \quad c_{i}^{j}=\left(1-\pi_{j}^{y}\right) y_{j} .
$$


We assume that both agents derive utility from the consumption of both final goods and leisure,

$$
u_{i}=u_{i}\left(c_{i}^{i}, c_{i}^{j}, f_{i}\right)
$$

The utility functions are assumed to be quasi concave.

Inserting (2)-(4) into (5) gives rise to the following maximization problem of the agents:

$$
\max _{l_{i}, a_{i}^{r}, d_{i}^{r}, a_{i}^{y}, d_{i}^{y}} u_{i}\left(\pi _ { i } ^ { y } F _ { i } \left(l_{i}, \pi_{i}^{r}+\left(1-\pi_{j}^{r}\right),\left(1-\pi_{j}^{y}\right) F_{j}\left(l_{j}, \pi_{j}^{r}+\left(1-\pi_{i}^{r}\right), f_{i}\right)\right.\right.
$$

Denote by $\delta_{i}=\left\{l_{i}, a_{i}^{r}, d_{i}^{r}, a_{i}^{y}, d_{i}^{y}\right\}$ the vector of instruments. A Nash equilibrium is a tupel $\left\{\delta_{1}^{N}, \delta_{j}^{N}\right\}$ such that both maximization problems are simultaneously fulfilled.

The first-order conditions are as follows:

$$
\begin{aligned}
\frac{d u_{i}}{d a_{i}^{r}} & =-\pi_{i}^{y} \frac{\partial u_{i}}{\partial c_{i}^{i}} \frac{\partial F_{i}}{\partial r_{i}} \frac{\partial \pi_{j}^{r}}{\partial a_{i}^{r}}+\left(1-\pi_{j}^{y}\right) \frac{\partial u_{i}}{\partial c_{i}^{j}} \frac{\partial F_{j}}{\partial r_{j}} \frac{\partial \pi_{j}^{r}}{\partial a_{i}^{r}}-\frac{\partial u_{i}}{\partial f_{i}} \gtreqless 0, \\
\frac{d u_{i}}{d d_{i}^{r}} & =\pi_{i}^{y} \frac{\partial u_{i}}{\partial c_{i}^{i}} \frac{\partial F_{i}}{\partial r_{i}} \frac{\partial \pi_{i}^{r}}{\partial d_{i}^{r}}-\left(1-\pi_{j}^{y}\right) \frac{\partial u_{i}}{\partial c_{i}^{j}} \frac{\partial F_{j}}{\partial r_{j}} \frac{\partial \pi_{i}^{r}}{\partial d_{i}^{r}}-\frac{\partial u_{i}}{\partial f_{i}} \gtreqless 0, \\
\frac{d u_{i}}{d a_{i}^{y}} & =-F_{j} \frac{\partial u_{i}}{\partial c_{i}^{j}} \frac{\partial \pi_{j}^{y}}{\partial a_{i}^{y}}-\frac{\partial u_{i}}{\partial f_{i}} \gtreqless 0, \\
\frac{d u_{i}}{d d_{i}^{y}} & =F_{i} \frac{\partial u_{i}}{\partial c_{i}^{i}} \frac{\partial \pi_{i}^{y}}{\partial d_{i}^{y}}-\frac{\partial u_{i}}{\partial f_{i}} \gtreqless 0, \\
\frac{d u_{i}}{d l_{i}} & =-\pi_{i}^{y} \frac{\partial u_{i}}{\partial c_{i}^{i}} \frac{\partial F_{i}}{\partial l_{i}}-\frac{\partial u_{i}}{\partial f_{i}} \gtreqless 0 .
\end{aligned}
$$

The first-order conditions have the following interpretation. (7): an increase in $a_{i}^{r}$ increases the quantity of resources agent $i$ possesses because he appropriates a larger share from agent $j$. This increases his production and decreases the production of the other agent. Given the fractions of the final good consumed, $\pi_{i}^{y},\left(1-\pi_{j}^{y}\right)$, the first term measures the increase in utility of agent $i$ due to an increase in production of good $i$ and the second term measures the decrease in utility of agent $i$ due to a decrease in production of good $j$. Both terms can be called the reallocation effect of resource appropriation. This effect need not be positive because an increase in the resources of an agent reduces the production of the other agent. In the extreme case where agent $i$ is very talented in appropriating goods $\left(\pi_{j}^{y}=0\right)$ but is not able to defend his own production $\left(\pi_{i}^{y}=0\right)$ every reallocation of resources is harmful for the agent. This is the filter function of the goods contest mentioned in the introduction: contrary to standard economic models with perfectly enforced property rights and developed markets an increase in the resource endowment of an agent need not increase the agent's utility.

The direct costs of an increase in appropriation of resources are equal to the marginal utility of leisure. Hence, for an interior solution the reallocation effect has to be equal to the marginal utility of leisure.

(8): The effect of an increase in defense of resources is similar to the effect of an increase in appropriation of resources. The first two terms measure the reallocation effect: an increase in $d_{i}^{r}$ increases the production of $\operatorname{good} i$ and reduces the production of good $j$. For an interior solution the net effect has to be equal to the marginal utility of leisure. 
(9), (10): An increase in activities to appropriate good $j$ or to defend good $i$ results in an increase in consumption of these goods. The total effect is the product of the marginal effect on the share of the good consumed and the total quantity of the good available, weighted by the marginal utility of this good. In an interior solution this effect has to be equal to the marginal utility of leisure. Assuming an interior solution we can calculate the optimal relationship between appropriative and defensive activities in the goods contest:

$$
\frac{\partial u_{i}}{\partial c_{i}^{j}} / \frac{\partial u_{i}}{\partial c_{i}^{i}}=-\frac{F_{i} \partial \pi_{i}^{y} / \partial d_{i}^{y}}{F_{j} \partial \pi_{j}^{y} / \partial a_{i}^{y}} .
$$

Appropriative and defensive activities are chosen in a way that the marginal rate of substitution between good $j$ and good $i$ is equal to the marginal rate of transformation between defensive and appropriative activities. This marginal rate of transformation is similar to the marginal rate of transformation from standard production theory. In the absence of property rights, appropriation and defense are productive activities. The productivity is defined by the contest technology which has a similar interpretation as a standard production technology. ${ }^{2}$ However, the marginal productivity of, for example, defense is equal to the marginal increase in the share that can be consumed times the total production. Please note that there exists no qualitative influence of the resource contest on the goods contest. An increase in consumption increases utility irrespective of the outcome of the resource contest. This shows that the direction of influence is asymmetric. This asymmetry between resource and goods contests can be demonstrated only if both contests are analyzed simultaneously.

(11): The optimal labor-supply decision has a standard interpretation. The first term measures the marginal increase in consumption resulting from an increase in labor supply, weighted by the marginal utility of consumption. In an interior equilibrium this effect has to be equal to the marginal utility of leisure.

Using (9) and (10), (7) and (8) can be simplified as follows:

$$
\begin{aligned}
& \frac{d u_{i}}{d a_{i}^{r}}=-\frac{\partial u_{i}}{\partial f_{i}}\left(\frac{\partial \pi_{j}^{r}}{\partial a_{i}^{r}}\left[\frac{\left(1-\pi_{j}^{y}\right) \frac{\partial F_{j}}{\partial r_{j}}}{F_{j} \frac{\partial \pi_{j}^{y}}{\partial a_{i}^{y}}}+\frac{\pi_{i}^{y} \frac{\partial F_{i}}{\partial r_{i}}}{F_{i} \frac{\partial \pi_{i}^{y}}{\partial d_{i}^{y}}}\right]+1\right) \leq 0, \\
& \frac{d u_{i}}{d d_{i}^{r}}=\frac{\partial u_{i}}{\partial f_{i}}\left(\frac{\partial \pi_{j}^{r}}{\partial d_{i}^{r}}\left[\frac{\left(1-\pi_{j}^{y}\right) \frac{\partial F_{j}}{\partial r_{j}}}{F_{j} \frac{\partial \pi_{j}^{y}}{\partial a_{i}^{y}}}+\frac{\pi_{i}^{y} \frac{\partial F_{i}}{\partial r_{i}}}{F_{i} \frac{\partial \pi_{i}^{y}}{\partial d_{i}^{y}}}\right]-1\right) \leq 0 .
\end{aligned}
$$

The economic intuition of these conditions is straightforward. An increase in appropriative or defensive activities by agent $i$ in the resource contest has three consequences. First, it decreases the total time available for leisure. This effect is measured by the term 1 . Second, it increases the amount of productive resources of agent $i$, which increases total production of good $i$. Third it reduces the amount of productive resources of agent $j$ which decreases the production of good $j$. The total effect of this reallocation on the utility of agent $i$ depends on the fractions of the goods he consumes as well as on the marginal utilities he derives from the two goods. This total effect is represented by the term in brackets, where the marginal utility of the goods are expressed in terms of marginal utility of leisure. As a consequence, if the weighted marginal utilities of both goods coincide, the term in brackets cancels, and the

\footnotetext{
$\overline{{ }^{2} \text { See Hirshleifer (2001) for details. }}$
} 
agents have no incentive to invest in the resource contest because the only remaining effect is the decrease in productive resources.

We can now present some general results.

Result 1. The agents will invest in the resource contest $\left(a_{i}^{r} \geq 0, d_{i}^{r}>0\right)$ if the appropriation of goods is impossible.

Proof: If the agents cannot appropriate the goods, $\pi_{i}^{y}=1$. In this case, (7) and (8) simplify to

$$
\begin{aligned}
& \frac{d u_{i}}{d a_{i}^{r}}=-\frac{\partial u_{i}}{\partial c_{i}^{i}} \frac{\partial F_{i}}{\partial r_{i}} \frac{\partial \pi_{j}^{r}}{\partial a_{i}^{r}}-\frac{\partial u_{i}}{\partial f_{i}}, \\
& \frac{d u_{i}}{d d_{i}^{r}}=\frac{\partial u_{i}}{\partial c_{i}^{i}} \frac{\partial F_{i}}{\partial r_{i}} \frac{\partial \pi_{i}^{r}}{\partial d_{i}^{r}}-\frac{\partial u_{i}}{\partial f_{i}} .
\end{aligned}
$$

The first term in both conditions is larger than zero, which is a necessary condition for an interior solution. Dividing by $\partial u_{i} / \partial f_{i}$ yields

$$
\begin{gathered}
-\frac{\partial u_{i} / \partial c_{i}^{i}}{\partial u_{i} / \partial f_{i}} \frac{\partial F_{i} \partial \pi_{j}^{y}}{\partial r_{i} \partial a_{i}^{r}} \gtreqless 0, \\
\frac{\partial u_{i} / \partial c_{i}^{i}}{\partial u_{i} / \partial f_{i}} \frac{\partial F_{i} \partial \pi_{i}^{r}}{\partial r_{i} \partial d_{i}^{r}} \gtreqless 0 .
\end{gathered}
$$

For $f_{i} \rightarrow 1$ the total production and consumption converges to zero. Hence, $f_{i}<1$ in equilibrium. Assume that $d_{i}^{r}=0$. In this case it is always optimal for agent $j$ to set $a_{j}^{r}>0$, which implies $\pi_{i}^{r}=0$, and in turn $\frac{\partial u_{i} / \partial c_{i}^{i}}{\partial u_{i} / \partial f_{i}}$ in (15) converges to $\infty$. Hence, $d_{i}^{r}>0$ in equilibrium. An analogous argument holds for $a_{i}^{r}$.

Result 2. The agents will not invest in the resource contest $\left(a_{i}^{r}=d_{i}^{r}=0\right)$ if they cannot defend against the appropriation of goods.

Proof: If the agents cannot defend against appropriation of the goods, $\pi_{i}^{y}=0$. In this case, (7) and (8) simplify to

$$
\begin{aligned}
\frac{d u_{i}}{d a_{i}^{r}} & =\frac{\partial u_{i}}{\partial c_{i}^{j}} \frac{\partial F_{j}}{\partial r_{j}} \frac{\partial \pi_{j}^{r}}{\partial a_{i}^{r}}-\frac{\partial u_{i}}{\partial f_{i}}, \\
\frac{d u_{i}}{d d_{i}^{r}} & =-\frac{\partial u_{i}}{\partial c_{i}^{j}} \frac{\partial F_{j}}{\partial r_{j}} \frac{\partial \pi_{i}^{r}}{\partial d_{i}^{r}}-\frac{\partial u_{i}}{\partial f_{i}} .
\end{aligned}
$$

In both cases the first term is smaller or equal to zero. The marginal utility of leisure is larger than zero, which gives the result.

Result 3. If both agents have the same production and appropriation functions they will not invest in the resource contest if $\pi_{i}^{y} \leq 1 / 2$. 
With equal production and contest functions the equilibrium has to be symmetric, $f_{i}=$ $f_{j}=f$, etc. Given this information, (7) and (8) reduce to

$$
\begin{aligned}
& \frac{d u_{i}}{d a_{i}^{r}}=\left(1-2 \pi^{y}\right) \frac{\partial u}{\partial c} \frac{\partial F}{\partial r} \frac{\partial \pi^{r}}{\partial a^{r}}-\frac{\partial u}{\partial f}, \\
& \frac{d u_{i}}{d d_{i}^{r}}=-\left(1-2 \pi^{y}\right) \frac{\partial u}{\partial c} \frac{\partial F}{\partial r} \frac{\partial \pi^{r}}{\partial d^{r}}-\frac{\partial u}{\partial f} .
\end{aligned}
$$

A sufficient condition for the conditions to be smaller than zero is that the first terms are smaller than zero. This is the case if $\pi^{y}<1 / 2$.

Result 1 characterizes the special case of resource conflicts analyzed in the literature. It comes as no surprise that this result reproduces the structure found in, for example, Grossman (2001) and Hirshleifer (1995). If the production of the own good cannot be challenged by the other agent, investments in the resource contest are the only means to improve utility beyond autarky. Given the assumptions of the model the agents would try to use this opportunity and appropriate some of the resource if the other agent does not invest in defense, which in turn implies that they will defend themselves against the appropriation by the other agent.

Result 2 shows that a positive consumption of the own good is necessary for an engagement in the resource contest. Agents are only interested in the appropriation of resources because it is a means to increase their final consumption. If the good produced is completely appropriated by the other agent there exist no incentives to improve the possession of resources. On the contrary, in a symmetric situation more resources would harm the agent because it would reduce the production of the other agent, which is completely appropriated.

Results 1 and 2 define two benchmark scenarios that illustrate the qualitative difference between resource and goods contests. This difference makes the optimal decision to invest in the resource contest much more complicated. An increase in resources is only attractive if a sufficient amount of the final good can be defended against appropriation. Otherwise it is more attractive to specialize in appropriation of the final good. Result 3 further clarifies this relationship. For the perfectly symmetric case the equilibrium fraction of the goods produced has to exceed $1 / 2$ in order to make it attractive to invest in the resource contest. However, this minimum fraction is only a necessary condition for positive investments. These investments are only positive if the marginal utility of consumption corrected by the net effect on consumption exceeds the marginal utility of leisure.

\section{A logarithmic Cobb-Douglas-Tullock economy}

The most popular functional specification in the literature on anarchy and contests is the CobbDouglas production and Tullock contest-success function economy. In order to simplify the exposition we will in addition assume that utility is logarithmic. However, it can be easily checked that the basic result does not depend on this simplification.

$$
\begin{aligned}
u_{i}\left(c_{i}^{i}, c_{i}^{j}, f_{i}\right) & =\ln c_{i}^{i}+\ln c_{i}^{j}+\ln f_{i}, \\
F_{i}\left(l_{i}, r_{i}\right) & =\sqrt{l_{i}} \cdot \sqrt{r_{i}}, \\
\pi_{i}^{r}\left(d_{i}^{r}, a_{j}^{r}\right) & =\frac{1}{1+\frac{a_{j}^{r}}{d_{i}^{r}}},
\end{aligned}
$$




$$
\pi_{i}^{y}\left(d_{i}^{y}, a_{j}^{y}, \theta\right)=\frac{1}{1+\theta \frac{a_{j}^{y}}{d_{i}^{y}}} .
$$

We know that a symmetric equilibrium exists because the maximization problems are perfectly symmetric. The first-order conditions characterizing a symmetric equilibrium are:

$$
\begin{aligned}
\frac{\partial u_{i}}{\partial a_{i}^{r}} & =-\frac{1}{2 l_{i}} \leq 0, \\
\frac{\partial u_{i}}{\partial d_{i}^{r}} & =-\frac{1}{2 l_{i}} \leq 0, \\
\frac{\partial u_{i}}{\partial a_{i}^{y}} & =-\frac{1}{2 l_{i}}+\frac{d_{i}^{y}}{a_{i}^{y}\left(d_{i}^{y}+\theta a_{i}^{y}\right)}, \\
\frac{\partial u_{i}}{\partial d_{i}^{y}} & =\frac{\left(d_{i}^{y}\right)^{2}+3 a_{i}^{y} d_{i}^{y} \theta+2 a_{i}^{y} \theta\left(a_{i}^{y}+a_{i}^{r}+d_{i}^{r}+f_{i}\right)}{2 d_{i}^{y}\left(-l_{i}\left(d_{i}^{y}+\theta a_{i}^{y}\right)\right)}, \\
\frac{\partial u_{i}}{\partial f_{i}} & =\frac{1}{f_{i}}-\frac{1}{2 l_{i}} .
\end{aligned}
$$

The first two conditions show that $d_{i}^{r}=a_{i}^{r}=0$, the agents prefer not to invest in the resource contest.

Result 4. In a Cobb-Douglas-Tullock economy with logarithmic utility functions the agents will not invest in the resource contest $\left(a_{i}^{r}=0, d_{i}^{r}=0\right)$.

Using this information and solving the remaining conditions yield

$$
f_{i}=\frac{2}{5}, \quad d_{i}^{y}=\frac{2}{5} \frac{\sqrt{\theta}}{1+\sqrt{\theta}}, \quad a_{i}^{y}=\frac{2}{5} \frac{1}{1+\sqrt{\theta}}, \quad a_{i}^{r}=d_{i}^{r}=0 .
$$

The solution has the following structure. First, $a_{i}^{y}=d_{i}^{y}(=1 / 5)$ if and only if $\theta=1$. Second, $\lim _{\theta \rightarrow 0} a_{i}^{y}=\lim _{\theta \rightarrow \infty} d_{i}^{y}=2 / 5$, and $\lim _{\theta \rightarrow \infty} a_{i}^{y}=\lim _{\theta \rightarrow 0} d_{i}^{y}=0$. Third, $\partial a_{i}^{y} / \partial \theta<0$ and $\partial a_{i}^{y} / \partial \theta>0$.

The solution shows that investments in the resource contest are an inferior instrument compared to investments in the goods contest. The mechanism explaining the result differs from the one that drives Results 1-3. In this example, both agents prefer a balanced consumption of both goods, which can only be guaranteed if the resource is equally distributed. With $\theta=1$ this results in an equal distribution of final consumption because

$$
\pi_{i}^{y}=1 /(1+\sqrt{\theta}), \quad \lim _{\theta \rightarrow 0} \pi_{i}^{y}=1, \quad \lim _{\theta \rightarrow \infty} \pi_{i}^{y}=0 .
$$

However, it could be argued that for small values of $\theta$ the final distribution of consumption becomes more and more unequal, which implies that the agents may want to use the resource contest to change the primary distribution in a way that reverses this effect. A reduction of the resources of one agent increases the production of the other agent. What is the implication of this change? If $\theta$ is very small the agent has a large fraction of this good already, which implies that the deviation from a balanced bundle of goods is getting larger. If $\theta$ is very large, 
the change in production has the right direction in principle. However, a large value of $\theta$ implies a small value of $\pi_{i}^{y}$, which implies that the fraction of the additional production that, can be appropriated is too small to more than compensate the loss (see Result 3). Hence, a redistribution of resources necessarily hurts the agent. This intuition can be made more precise if one calculates the value of the indirect utility function (that is a function of $\theta$ ). Denoting the indirect utility function by $w_{i}(\theta)$, we get:

$$
w_{i}(\theta)=\ln \left(\frac{2}{25(1+\sqrt{\theta})}\right)+\ln \left(\frac{\sqrt{\theta}}{1+\sqrt{\theta}}\right) .
$$

It is easy to check that the maximum of the indirect utility function is reached at $\theta=1$. As can be expected, if both agents are equally decisive in the goods contest the resulting allocation of goods is equal. Any asymmetry in the decisiveness creates an unequal distribution of the goods, which reduces utility.

\section{Conclusions}

In this paper we have analyzed the relationship between resource and goods contests in a model where agents can simultaneously invest in both contests. Two main findings result from the analysis. First, the simultaneous analysis of both contests reveals a structural difference between resource and goods contests that does not emerge from their separate analysis. The incentives to engage in a resource contest are determined by the structure of the goods contest. Success in the resource contest can be counterproductive if it is not accompanied by success in the goods contest. Second, for the most common functional specification with Tullock contest and Cobb-Douglas production functions it turns out that the agents will restrict attention to the goods contest investing nothing in the appropriation of resources.

Acknowledgements I thank Herschel Grossman for helpful comments on this paper. The kind hospitality of Brown University where most of the paper has been written is gratefully acknowledged. Research on this paper has been supported by the German Research Foundation.

\section{References}

Bush, W.C., \& Mayer, L.S. (1974). Some implications of anarchy for the distribution of property. Journal of Economic Theory, 8(4), 401-412.

de Meta, D., \& Gould, J.R. (1992). The social efficiency of private decisions to enforce property rights. Journal of Political Economy, 100(3), 561-580.

Grossman, H. (2001). The creation of effective property rights. American Economic Review, 91, 347-352.

Grossman, H. (2002). Make us a king: Anarchy, predation, and the State. Forthcoming: European Journal of Political Economy.

Grossman, H., \& Kim, M. (1995). Swords or plowshares? A theory of the security of claims to property. Journal of Political Economy, 103(6), 1275-1288.

Hirshleifer, J. (1995). Anarchy and its breakdown. Journal of Political Economy, 103(1), $26-52$.

Hirshleifer, J. (2001). The dark side of the force. In J. Hirshleifer (Ed.), The dark side of the force (pp. 7-22). Cambridge: Cambridge University Press.

Lancaster, K. (1966). A new approach to consumer theory. Journal of Political Economy, 74, 132-157.

Skaperdas, S. (1992). Cooperation, conflict, and power in the absence of property rights. American Economic Review, 82(4), 720-739.

Skaperdas, S., \& Syropoulos, C. (1997). The distribution of income in the presence of appropriative activities. Economica, 64(253), 101-117. 